\title{
INFLUENCE DE LA MESURE CHIMIQUE DU DÉBIT SUR LES INVERTÉBRÉS D'UN RUISSEAU DE MONTAGNE
}

\author{
par H. DÉcamps ${ }^{1}$ et J. M. ElliotT ${ }^{2}$.
}

\section{Introduction.}

Les méthodes de mesure chimique du débit sont couramment utilisées dans les ruisseaux turbulents aux eaux fortement brassées. C'est le cas de la plupart des cours d'eau de montagne. Souvent, pour ces derniers, seule l'introduction puis le dosage de substances chimiques permettent une estimation précise du débit.

De semblables perturbations - pesticides, toxiques - peuvent entraîner l'apparition d'une dérive catastrophique (Waters 1972). Il était donc intéressant d'observer l'effet d'une mesure chimique de débit sur la dérive des invertébrés.

\section{Ruisseau étudié.}

L'expérience a été réalisée au mois d'août, à $2180 \mathrm{~m}$ d'altitude sur le ruisseau d'Estaragne dans les Pyrénées centrales. Les caractéristiques physico-chimiques et biologiques de ce ruisseau sont précisées ailleurs (Décamps 1967, Décamps et Lavandier 1970). Au niveau étudié, l'Estaragne coule en prairie subalpine. Le débit atteignait $155 \mathrm{l} / \mathrm{sec}$ au moment de l'expérience. Les conditions d'écoulement aux points d'échantillonnage sont résumées au tableau 1.

\section{Méthodes et techniques d'échantillonnage.}

1. Mesure chimique du débit. - Le point d'injection étant choisi, la mesure du débit est effectuée en deux temps. Dans un premier temps, de la fluorescéine est introduite dans le ruisseau pour déterminer en quel transect les eaux sont correctement brassées vers l'aval. Dans un second temps, une quantité connue de bichromate est injectée régulièrement pendant 30 minutes environ.

1. Laboratoire d'Hydrobiologie, Université Paul-Sabatier, Toulouse.

2. Freshwater Biological Association, Ambleside, Westmorland. 
Ce bichromate est ensuite dosé au laboratoire sur plusieurs échantillons recueillis au transect préalablement déterminé.

Dans cette expérience, la solution-mère de bichromate $(500 \mathrm{~g}$ dans 40 litres d'eau) étant injectée à raison de $0,01 \mathrm{l} / \mathrm{sec}$, la concentration de bichromate dans l'eau de l'Estaragne (débit $155 \mathrm{l} /$ sec. $=0,155 \mathrm{~m}^{3} / \mathrm{sec} .=279 \mathrm{~m}^{3} / 30 \mathrm{mn}$ ) atteignait $0,8 \mathrm{mg} / \mathrm{l}$.

2. Echantillonnage de la dérive. - Des filets de surface (décrits en détail par Elurotr 1967, 1970) ont été utilisés pour échantillonner la dérive. Le filet amont était placé immédiatement en amont du point d'injection de la fluorescéine et du bichromate, le filet aval était placé à l'aval (environ $200 \mathrm{~m}$ ) du point d'injection. Les trois échantillons pris avec le filet amont servaient de contrôle pendant l'introduction de la fluorescéine et du bichromate; chacun d'eux durait 30 minutes (tableau 2). Quatre échantillons étaient pris avec le filet aval : le premier sur 30 minutes avant toute introduction, le deuxième sur 35 minutes pendant le passage de la fluorescéine, le troisième sur 30 minutes pendant l'introduction du bichromate, et le quatrième sur 30 minutes après que le bichromate ait passé le point d'échantillonnage (tableau 2). Le deuxième échantillon a duré 5 minutes de plus que les autres. Cependant, par suite d'un délai entre le point d'injection et le filet aval, 15 minutes se sont écoulées avant le passage de la fluorescéine au niveau du filet. La durée effective d'échantillonnage en présence de fluorescéine n'a donc été que de 20 minutes.

Un micromoulinet Ott a été utilisé pour mesurer les vitesses du courant à l'ouverture des filets. Ces mesures ont servi à estimer le volume d'eau échantillonné par chaque filet. Les vitesses de courant et volumes d'eau échantillonnés sont résumés dans le tableau 1; les périodes d'échantillonnage et le traitement de l'eau sont résumés dans le tableau 2 .

Tableau 1. - Conditions d'écoulement aux points d'échantillonnage : moyenne $\pm 95 \%$ des limites de confiance.

Filet amont

Filet aval

Vitesse du courant (en $\mathrm{m} / \mathrm{sec}$.)

$0,60( \pm 0,20)$

$0,56( \pm 0,15)$

Volume d'eau filtré à chaque échantillon de $30 \mathrm{mn}$ (en $\mathrm{m}^{3}$ )

$43,47( \pm 14,61) \quad 35,04( \pm 7,54)$

Volume d'eau filtré dans

l'échantillon de $35 \mathrm{mn}\left(\mathrm{en} \mathrm{m}^{3}\right)$

$40,88( \pm 8,80)$ 


\section{Résultats.}

Les nombres obtenus dans les filets amont et aval figurent aux tableaux 2 et 3.

Un test $\chi^{2}$ de conformité avec une distribution de Poisson (voir Elliotr 1970, section 4.1.3.) a été appliqué aux échantillons du filet amont. La conformité avec une distribution de Poisson étant acceptée $(P>0,05)$, il n'est pas apparu de différence significative entre les nombres totaux d'invertébrés benthiques de chaque échantillon, ni entre les trois comptages pour chaque taxon. Un résultat semblable a été obtenu en appliquant un test $\chi^{2}$ aux échantillons 1 et $4 \mathrm{du}$ filet aval. Par suite, un simple test de comparaison de deux séries de Poisson (voir Ellootr 1971, p. 104) a permis de comparer la moyenne des captures des échantillons 1 et 4 avec les captures obtenues pendant lintroduction de fluorescéine (échantillon 2) et de bichromate (échantillon 3). La dérive augmentait pour plusieurs taxa dans les échantillons 2 et 3 (par ex. Baetis cf. alpinus, larves de Chironomides), mais aucune de ces augmentations n'était significative $(P>0,05)$. Seuls, les accroissements du nombre total d'invertébrés dans la dérive (c.a.d. 86 dans l'échantillon 2, et 80 dans l'échantillon 3) étaient significatifs ( $\mathrm{P}<0,01$ pour la fluorescéine, $\mathrm{P}<0,05$ pour le bichromate).

Par suite, un léger accroissement de la dérive existait quand la fluorescéine ou le bichromate étaient introduits dans le ruisseau. Mais cet accroissement n'était statistiquement significatif que pour les nombres totaux, et non pour les taxa pris séparément.

\section{Discussion.}

Quoique statistiquement significative, l'augmentation de la dérive totale apparaît relativement faible. Une importante dérive catastrophique sur les 200 mètres entre le point d'injection et le filet aval aurait entraîné une augmentation importante des captures. Les invertébrés capturés dans le filet aval semblent donc ne provenir que des quelques mètres situés immédiatement au-dessus. Sur l'ensemble de la section de $200 \mathrm{~m}$, les invertébrés en dérive ont probablement effectué un retour rapide au substrat. Ils ne se retrouvent pas dans le filet aval. De plus, l'action du bichromate sur la dérive cesse dès que l'injection est terminée : l'échantillon $4 \mathrm{du}$ filet aval est remarquablement semblable à l'échantillon 1 de ce même filet. Les déterminations chimiques de débit ont donc un effet modéré sur la dérive des invertébrés benthiques, dans la mesure où de faibles quantités de produits sont injectées dans le cours d'eau.

En l'absence de tout traitement de l'eau, les nombres d'invertébrés benthiques récoltés dans les filets amont (échantillons 1, 2, 


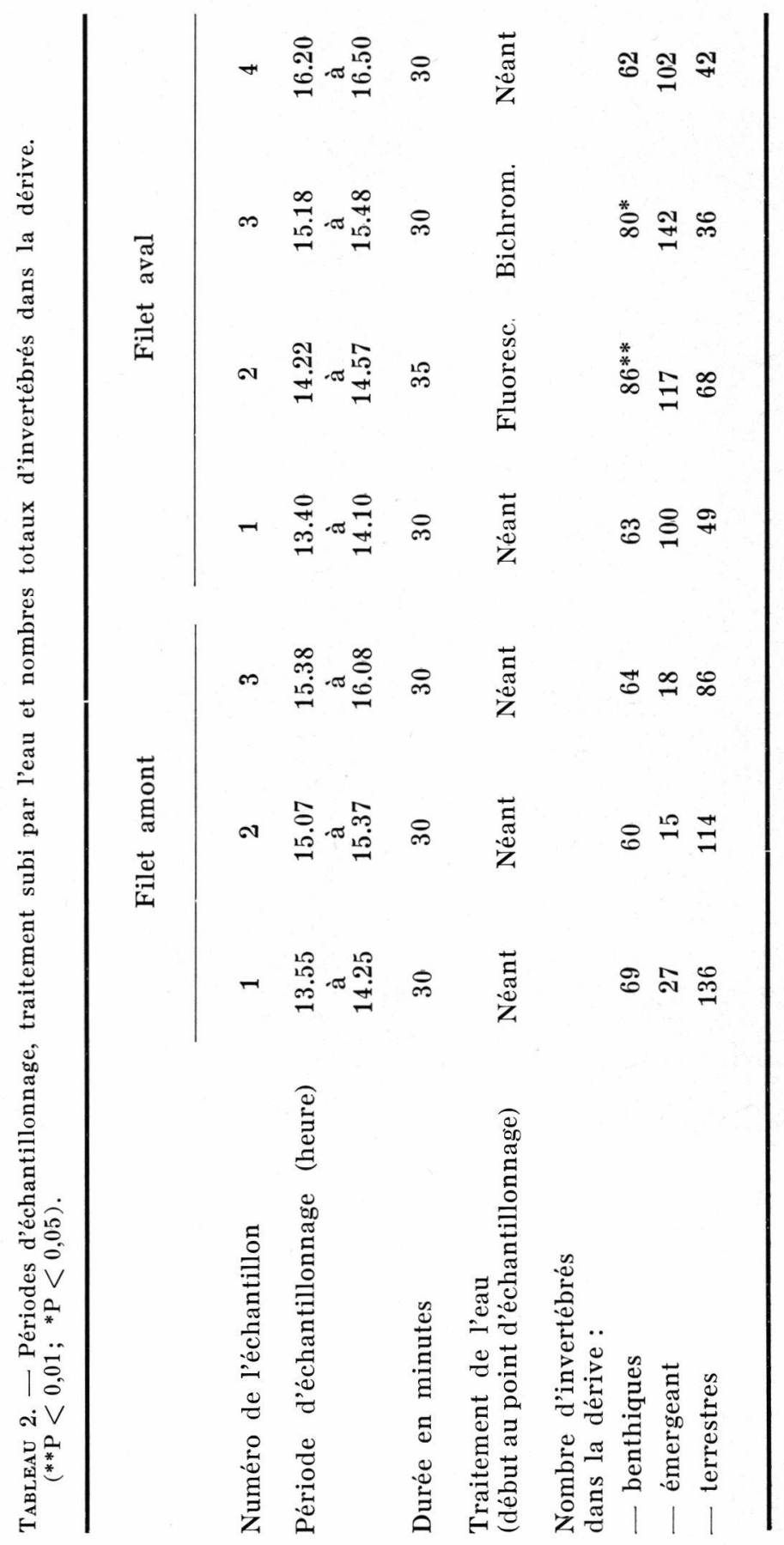




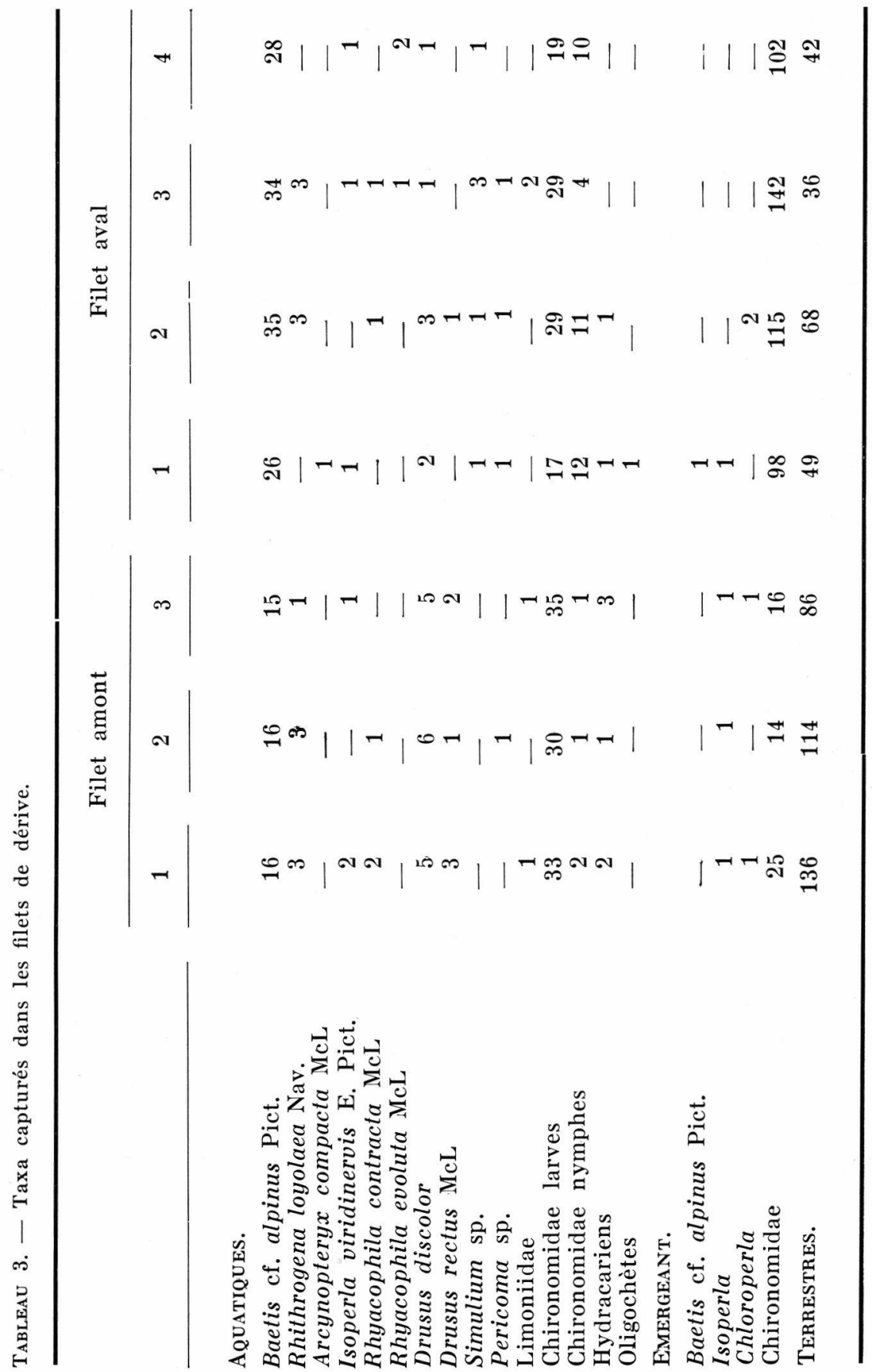


3) et aval (échantillons 1 et 4) ont présenté peu de variations à cette période de la journée. Il en est de même des taxa individuels, notamment de Baetis ef. alpinus, de Drusus discolor et des larves de Chironomides. Par ailleurs, on doit souligner les faibles quantités de fluorescéine et de bichromate $(0,8 \mathrm{mg} / \mathrm{l})$ utilisées, ainsi que la brièveté des périodes d'injection. Dans ces conditions, les différences constatées indiquent la grande sensibilité des invertébrés benthiques aux perturbations subies par le ruisseau.

Remerciements. - Nous remercions MM. Pourciel et Trivellato pour leur aide sur le terrain, et MM. Berthélemy et Thomas pour leurs déterminations des Plécoptères et des Ephémères.

\section{RESUME}

Les variations dans la dérive des invertébrés ont été observées au cours d'une expérience de mesure chimique du débit dans un ruisseau de montagne. Un léger accroissement de dérive existait quand de la fluorescéine ou du bichromate étaient introduits dans le ruisseau. Cet accroissement n'était statistiquement significatif que pour les nombres totaux et non pour les taxa pris séparément.

\section{THE EFFECT OF A CHEMUCAL METHOD FOR GAUGING DISCHARGE ON THE INVERTEBRATES OF A MOUNTAIN RIVER}

Variations in invertebrate drift were studied during the gauging of discharge in a mountain river (constant rate injection method with dichromate during gauging, and fluorescein in the preliminary trial). There was a slight increase in the numbers in the drift when the fluorescein or dichromate was introduced into the river, but this increase was only statistically significant for total numbers (Table 2), not for individual taxa (Table 3 ).

\section{TRAVAOX GITÉS}

Décamps (H.). 1967. - Ecologie des Trichoptères de la vallée d'Aure (Hautes-Pyrénées). Annls Limnol., 3 : 399-577.

Décamps (H.) et Lavandier (P.). 1970 (1972). - La faune d'un torrent de haute montagne dans les Pyrénées. In KaJaK (Z.) et HillBricht-Ilkowska (A.) : Productivity Problems of Freshwaters : 875-881.

Ellioti (J. M.). 1967. - Invertebrate drift in a Dartmoor stream. Arch. Hydrobiol., 63 (2) : 202-237.

ElLIOTT (J. M.). 1970. - Methods of sampling invertebrate drift in running water. Annls Limnol., 6 : 133-159.

Elllot (J.M.). 1971. - Some methods for the statistical analysis of samples of benthic invertebrates. Scient. Publs Freshwat. biol. Ass., $\mathrm{n}^{\circ}$ 25: 144 p.

Waters (T. F.). 1972. - The drift of stream insects. Ann. Rev. Entomol., 17 : $253-272$. 